\title{
Microvessel density in Prostatic Lesions : Relevance to prognosis
}

\author{
Upadhyaya $\mathrm{P}^{1}$, Agarwal $\mathrm{CS}^{2}$, Karak $\mathrm{AK}^{3}$,Sinha $\mathrm{AK}^{1}$, Karki S ${ }^{1}$, Dhakal S1, \\ Khadka $\mathrm{D}^{1}$ \\ ${ }^{I}$ Department of Pathology, B.P Koirala Institute of Health Sciences, Dharan, Nepal. \\ ${ }^{2}$ Department of Surgery B.P Koirala Institute of Health Sciences, Dharan, Nepal. \\ ${ }^{3}$ Department of Pathology, All India Institute of Medical Sciences, Delhi, India.
}

\section{Keywords:}

Angiogenesis;

Microvessel Density;

Prostate;

Prostatic Carcinoma;

Gleason's Score

\begin{abstract}
Background: Angiogenesis is required for growth and metastasis of tumor tissue. Quantization of angiogenesis by calculating the microvessel density can be done in histopathology specimens with the help of immunochemistry. In this study we used anti CD 34 antibody to highlight the endothelial cells and thus calculate microvessel density. Most studies have shown a positive correlation of microvessel density with increasing pathological grade and have also shown microvessel density as an independent predictor of cancer progression and survival. The present study was to find out the microvessel density in benign and malignant lesions of prostate and also to correlate the vascularity with increasing grade of cancer.
\end{abstract}

Materials and methods: Sixty five prostatic biopsies were evaluated for microvessel density using CD34 monoclonal antibody. Comparison was done between BPH and Carcinoma Prostate. MVD was correlated with Gleason's score, weight of specimen and increasing age of patient. Effect of prostatitis on Microvessel density was studied.

Result: Microvessel density was significantly higher in carcinoma prostate than in Benign Prostatic Hyperplasia. There was positive correlation of Microvessel density with increasing Gleason's score. Microvessel was significantly increased in patients having symptoms for more than a year and also with biopsies revealing prostatitis. However, there was no significant correlation between Microvessel density and weight of specimen or increasing age.

Conclusion: Since Microvessel density was found to be significantly higher in Prostatic Carcinoma and it showed positive correlation with Gleason's score it can be added as one of the indicators for predicting the disease outcome.

\section{INTRODUCTION}

Angiogenesis is required for growth and metastasis of tumor tissue. Quantization of angiogenesis by calculating the Microvessel density (MVD) can be done in histopathology

\section{Correspondence:}

Dr. Paricha Upadhyaya, MBBS, MD, Department of Pathology

B.P Koirala Institute of Health Sciences, Dharan, Nepal

Email : paricha7@yahoo.com specimens with the help of immunochemistry (IHC) using antibodies like CD 34, CD 31 and factor VII against endothelial antigens. ${ }^{1}$ Anti CD 34 antibody is used to highlight the endothelial cells and thus calculate MVD. This method can be done if IHC facilities are available however the values received can vary subjectively. The other method is imagecytometry which is relatively expensive, but gives a standardized report. ${ }^{2}$ 
Transurethral resection of prostate for benign prostatic hyperlasia and needle biopsies for diagnosis of carcinoma is the commonly received specimens in the histopathology department for disease of the prostate.The importance of these biopsies is especially to diagnose carcinoma and to grade them or detect early lesions such as prostatic intraepithelial neoplasia (PIN) which is considered a precancerous lesion. ${ }^{3}$ Diagnosis of carcinoma can also be aided by preoperative prostatic specific antigen (PSA) estimation and use of imaging studies coupled with clinical examination while biopsy remains the gold standard in confirming a carcinoma. ${ }^{3}$

Besides reporting cancer and grading it, there are number of other features, which add to the predictive value, which may be included in the routine reporting of cancers; one such feature is estimation of MVD. Most studies have shown a positive correlation of MVD with increasing pathological grade $^{4,5}$, while some other studies have found out that MVD is an independent predictor of cancer progression and survival. ${ }^{6,7}$ The present study was taken up to see if there was a significant difference in MVD in benign and malignant lesions of prostate and also to correlate the vascularity with increasing grade of cancer.

\section{MATERIAL AND METHODS}

This was a retrospective cross-sectional descriptive study conducted at BP Koirala Institute of Health Sciences permission from the Ethics comittiee was obtained. All the specimen of prostate submitted to department of Pathology within a year was included in the study. Besides 24 cases with prostatic carcinoma diagnosed over a period of past seven years was also included. Specimen were processed routinely and paraffin embedded specimens ( 3 to $5 \mathrm{~mm}$ ) thick were stained with hematoxylin and eosin. Slides were examined under low power light microscopy to identify the tumoral areas.

For immunohistochemistry the tissue-blocks bearing significant amounts of tumor tissue were then re-cut and mounted on slides. These sections were deparaffinized and hydrated. Endogenous peroxidase was inhibited wit 3\% hydrogen peroxide. The avidin biotin-peroxidase technique was used for imuunohistochemical analysis.

Microvascular density was detected using the commercially available CD34 monoclonal antibody. The area of maximal CD34 expression was identified in tumor tissue. Within tumor zones, in the area of maximal angiogenesis, microvessels were counted on a 200x magnification field $\left(1.05 \mathrm{~mm}^{2}\right.$ Nikon: E600). Any dark staining endothelial cell or cell cluster clearly separate from adjacent structures was considered a single vessel. The results of the count was expressed in terms of an average microvessel count per square millimeter of tissue area $\left(\mathrm{vv} / \mathrm{mm}^{2}\right)$ and expressed as microvessel density (MVD).

\section{Statistical Analysis}

The obtained data were compiled in excel file and analyzed using statistical package for social science. Descriptive statistics were calculated like percentage, proportion, mean and standard deviation, diagrammatic presentation. For inferential statistics chi-square t-test and spearman's rho correlation coefficient was applied to find out the association and correlation between inter and intra group. For this purpose $95 \%$ CI was taken where $\mathrm{p}=0.05$.

\section{RESULTS}

A total of 65 prostatic biopsies were in this study out of which 41 cases were of nodular hyperplasia and 24 prostatic carcinomas. The age ranged from 35 years to 88 years, for cases of nodular hyperplasia prostate with a mean of 68 years. The youngest carcinoma of prostate was 55 years old and the oldest was 85 years of age with a mean of 69 years.

\section{Microvessel density (MVD)}

The overall microvessel density in our study ranged from $25 \mathrm{ti}+\mathrm{o} 285 \mathrm{vv} / \mathrm{mm}^{2}$. In BPH the MVD ranged from 25 to 127. The mean was 94 vessels $/ \mathrm{mm}^{2}$. In carcinoma the MVD ranged from 115 to 285 and the mean was $171.5 \mathrm{vv} \mathrm{m^{2 }}$. The mean MVD of BPH was 94 and the mean of carcinoma was 171.5. Comparison of MVD of BPH with carcinoma prostate showed that microvessel density was significantly higher in carcinoma prostate where $\mathrm{t}=10.824$, and $\mathrm{p}$ value was $<0.001$.

Gleason's score is used as a marker of predictive outcome in carcinoma prostate and to fulfil the second objective of this study MVD was compared with Gleason's score. The results showed a positive correlation of MVD with increasing Gleason's score where rho $=0.449$ and with a $\mathrm{p}$ value of 0.028 . MVD was found to be significantly increased in patients having symptoms for more than a year with a $\mathrm{p}$ value of 0.002 . Microvessel density of prostate biopsies, which showed evidence of inflammation (prostatitis), was compared with the biopsies that did not show any inflammation. Statistical analysis showed that there was significant increase in MVD in prostate biopsies revealing prostatitis with a $p$ value of 0.001 and $t=3.34$.

\section{DISCUSSION}

Microvessel density, as quantified in immunohistochemistry stained tissue sections, has proven to add prognostic information in a variety of tumour.8 In this study comparison of MVD of BPH with carcinoma prostate showed that microvessel density was significantly higher in carcinoma prostate where $\mathrm{t}=10.824$, and $\mathrm{p}$ value was $<0.001$. Few studies have been done comparing presence of MVD in benign prostatic hyperplasia and prostatic carcinoma. 
Chetinkaya et al studied MVD levels in BPH and advanced prostatic carcinoma. Their study showed no significant relation of MVD in BPH whereas there was positive relation of MVD in prostatic carcinoma. ${ }^{9}$

Angiogenesis is regarded as a prognostic marker in Prostatic Carcinoma. The mean blood vessel count is higher in tumors with metastases than those without metastasis and most studies but not all show a correlation with pathologic stage. MVD appears to be an independent predictor of cancer progression in some studies. The cumulative data suggest that increased MVD contributes to the extra prostatic spread of adenocarcinoma, perhaps by facilitating microvascular invasion. ${ }^{10}$

Tumors stimulate angiogenesis by directly secreting angiogenic substances or by activating and releasing angiogenic compounds stored within the extracellular matrix. Tumor-associated vascularity also can be enhanced by angiogenic substances secreted by tumor infiltrating lymphocytes, macrophages, or mast cells. The end result is the activation of nearby endothelial cells, which respond by expressing a cell autonomous pattern of behavior that culminates in the formation of new vessels. ${ }^{11}$

In this study, we compared microvessel density in Benign Prostatic Hyperplasia and Prostate Cancer using CD34 and analyzed its relevance to prognosis.

Gleason's score is widely used grading system for Prostate Carcinoma. Positive correlation was observed between Gleason's score and MVD with $r h o=0.449$ and $\mathrm{p}$ value of 0.028 showing prognostic role of MVD. Bono et al performed study on MVD in Prostatic carcinoma using CD34 which showed positive relation between MVD and pathological stage and also with Gleason's score. ${ }^{1}$ de la Taille et al showed direct association of MVD with stage of Prostate Carcinoma using CD 34 and 31.Of the cases with organ confined disease pT2 $10 \%$ had CD 34 of 105 or more vs. $29 \%$ of patients with pT3 disease. They also showed association of MVD with an increased likelihood of PSA recurrence; the percentage of patients with an MVD/CD 34 of 105 or more was $60 \%$ vs. $23 \%$ for patient with MVD less than $105 .{ }^{11}$

However, studies conducted by Silbermann MA et al and Rubin MA et al failed to show statistical association between microvessel density and stage or Gleason Score..$^{12,13}$

In a study done by Weidner $\mathrm{N}$ et al in University of California, San Francisco, it was shown that tumor angiogenesis correlates with metastasis in invasive prostate carcinoma. Within invasive prostate carcinomas from patients having metastasis the mean microvessel count was 76.8 per $\times 200$ field. For those carcinomas from patients without metastases the mean microvessel count was 39.2 per x200 field. The Wilcoxon rank sum test showed these differences to be statistically significant. ${ }^{14}$

MVD was found to be significantly increased in patients having symptoms for more than a year with $\mathrm{p}$ value of 0.002 . Statistical analysis showed that there was significant increase in MVD in prostate biopsies when there was presence of inflammation with $\mathrm{t}=3.34$ and $\mathrm{p}$ value of 0.001 . However, there was no significant correlation of MVD with weight of biopsy specimen and increasing age of the patient. Our results are consistent with the study of Bettencourt et al who performed assessment of angiogenesis as a prognostic marker for prostate cancer recurrence using CD34. Mean microvessel density count did not vary significantly with age. There was a significant association between the count and nuclear grade, Gleason sum and pathological stage. Cox survival analysis shows that microvessel density is significantly related to time to recurrence when considered as a continuous variable as well as dichotomous variable. The 5-year recurrence-free survival was significantly higher for patients with a count less than $90(71 \%)$ than for those with a count 90 or greater $(51 \%)(\mathrm{p}=0.006)$. The 5 -year recurrence-free survival was also significantly different when microvessel density was used as a continuous variable $(p=0.02)$. Controlling for stage, Gleason sum, race and nuclear grade, microvessel density remained significant in predicting recurrence $(\mathrm{p}=0.03) .{ }^{15}$

Benign prostatic hyperplasia and prostate cancer are among the most common diseases of the prostate gland and represent significant burdens for patients and healthcare systems in many countries. Our results, which are in coherent with those stated in literature highlights the prognostic significance of MVD assessment in histological evaluation of prostate biopsy.

\section{CONCLUSION}

Our data showed that angiogenesis was significantly higher in carcinoma than in BPH. Increased MVD was significantly associated with high-grade carcinoma. Therefore, vascular density can be added as one of the indicators for predicting the disease outcome. CD34 is suitable marker for the imuunohistochemical visualization of microvessel in benign and malignant prostatic tissue. These results expand our understanding about the role of angiogenesis in the growth of tumors and point out that angiogenesis are required for tumor sustainability as it was significantly associated with the duration of the disease. Furthermore, it also supports the role of antiangiogenic treatment in tumors of the prostate, however, in combination with other treatment modalities.

\section{REFERENCES}

1. Bono A, Celato N, Cova V, Salvadore M, Chinetti S, Novario R. Microvessel density in prostate carcinoma. Prostate Cancer Prostatic Dis 2002;5:123-7. Crossref 
2. Choi WWL, Lewis MM, Lawson D, Yin-Goen Q, Birdsong GG, Cotsonis $\mathrm{G}$ a, et al. Angiogenic and lymphangiogenic microvessel density in breast carcinoma: correlation with clinicopathologic parameters and VEGF-family gene expression. Mod Pathol. 2005;18:143-52. Crossref

3. Montironi R, Vela Navarrete R, Lopez-Beltran A, Mazzucchelli R, Mikuz G, Bono A V. Histopathology reporting of prostate needle biopsies. 2005 Update. Virchows Arch. 2006;449:1-13. Crossref

4. Lo J, Kerns B, Amling C, Robertson C, Layfield L. Correlation of DNA ploidy and histologic diagnosis from prostate core-needle biopsies: is DNA plody more sensitive than histology for the diagnosis of carcinoma in small specimens? J Surg Oncol. 1996;63:41-5. Crossref

5. Takai K, Goellner J, Katzmann JA A. Static image and flow DNA cytometry and prostatic adenocarcinoma: studies of needle biopsy and radical prostatectomy specimens. J Urol Pathol. 1994;2:3948.

6. Bostwick D, Wheeler T, Blute M. Optimized Microvessel density analysis improves prediction of cancer stage from prostate needle biopsies. Urology. 1996;48:47-57. Crossref

7. Montironi R, Magi- Galluzzi C, Diamanti L, Taborro R, Scarpelli M, Pisani E. Prostatic Intraepithelial Neoplasia: qualitative and quantitative analysis of the blood capillary architecture on this tissue sections. Pathol Res Pr. 1993;189:542-8. Crossref

8. Wester K, Ranefall P, Bengtsson E, Busch C, Malmström PU. Automatic quantification of microvessel density in urinary bladder carcinoma. Br J Cancer. 1999;81:1363-70. Crossref
9. Cetinkaya M, Gunce S, Ulusoy E, Aksoy F, Yildiz O, Adsan O, et al. Relationship between Prostate Specific Antigen Density, Microvessel Density and Prostatic Volume in Benign Prostatic Hyperplasia and Advanced Prostatic Carcinoma. Int Urol Nephrol. 1998;30:581-5. Crossref

10. Montironi R, Mazzucchellia R, Scattonib V, Bostwicke D. Pathological Findings in TRUS Prostatic Biopsy-Diagnostic, Prognostic and Therapeutic Importance. Eur Urol Suppl. 2002;1:6075. Crossref

11. de la Taille A, Katz AE, Bagiella E, Buttyan R, Sharir S, Olsson CA. Microvessel Density as a Predictor of PSA Recurrence After Radical Prostatectomy A Comparison of CD34 and CD31. Anat Pathol. 2000;555-62.

12. Silberman M, Partin A, Veltri R. Tumor angiogenesis correlates with progression after radical prostatectomy but not with pathologic stage in Gleason sum 5-7 adenocarcinoma of the prostate. Cancer. 1997;79:772-9. Crossref

13. Rubin M, Buyyounouski M, Bagiella E. Microvessel density in prostate cancer: lack of association with tumor grade, pathologic stage and clinical outcome. Urology. 1999;53:543-7. Crossref

14. Weidner N, Carroll PR, Flax J, Blumenfeld W, Folkman J. Tumor angiogenesis correlates with metastasis in invasive prostate carcinoma. Am J Pathol. 1993;143:401-9. Crossref

15. Bettencourt MC, Bauer JJ, Sesterhenn IA, Connelly RR, Moul JW. CD34 immunohistochemical assessment of angiogenesis as a prognostic marker for prostate cancer recurrence after radical prostatectomy. J Urol. 1998;160:459-65. Crossref. Crossref 